\title{
ERRATUM
}

\section{Combined STAT3 and BCR-ABL1 inhibition induces synthetic lethality in therapy-resistant chronic myeloid leukemia}

AM Eiring, BDG Page, IL Kraft, CC Mason, NA Vellore, D Resetca, MS Zabriskie, TY Zhang, JS Khorashad, AJ Engar, KR Reynolds, DJ Anderson, A Senina, AD Pomicter, CC Arpin, S Ahmad, WL Heaton, SK Tantravahi, A Todic, R Colaguori, R Moriggl, DJ Wilson, R Baron, T O'Hare, PT Gunning and MW Deininger

Leukemia (2017) 31, 1253-1254; doi:10.1038/leu.2017.14

Correction to: Leukemia (2015) 29, 586-597; doi:10.1038/ leu.2014.245

It has come to our attention that a production error made by the Journal resulted in duplication of the immunoblot image from Figure $2 a$ into the analogous position in Figure $2 b$, replacing the correct image for that panel. The complete, correct version of Figure 2 as originally submitted by the authors appears below. We thank the Journal for providing this correction. 


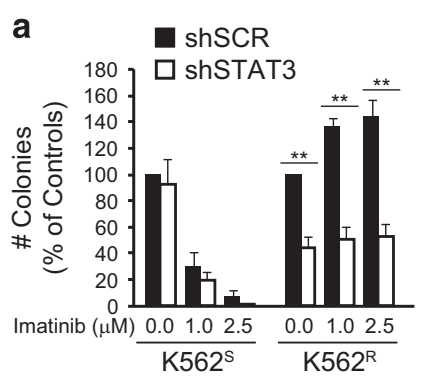

C

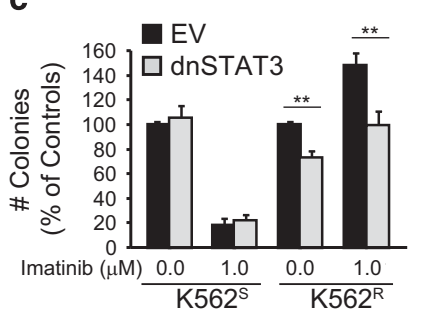

e

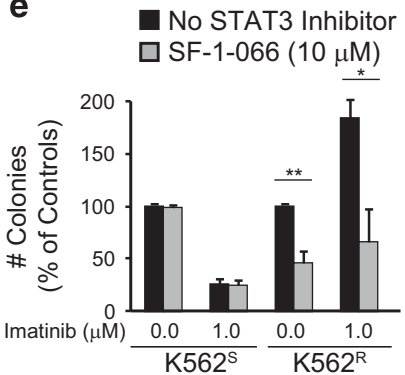

K562

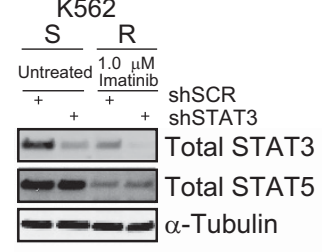

b

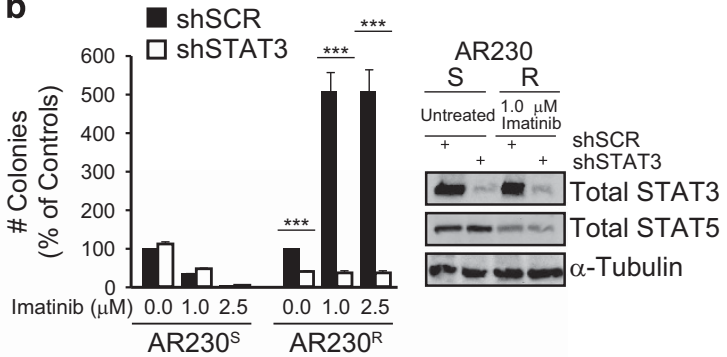

d
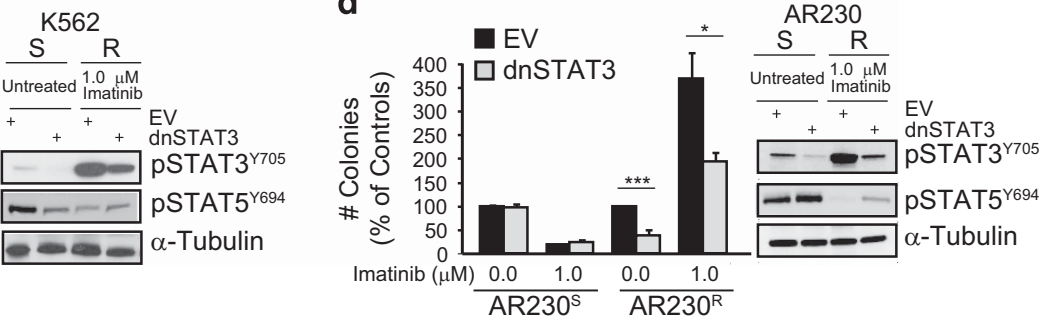

f

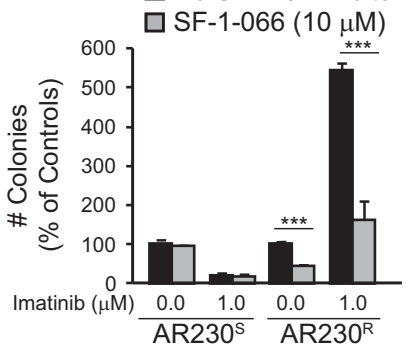

9

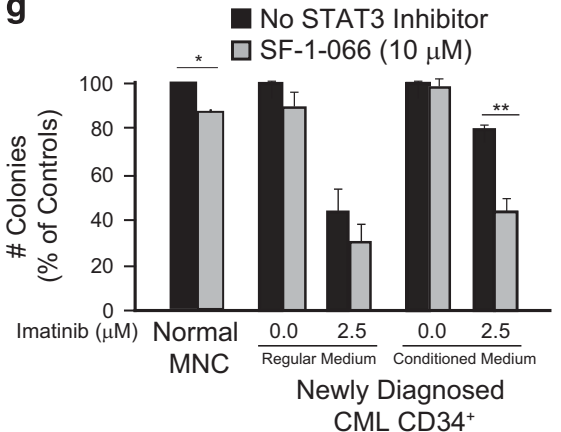

Figure 2. Inhibition of STAT3 reduces colony formation by TKI-resistant CML cells. (a, b) TKI-resistant CML cell lines were retrovirally transduced with shRNA targeting STAT3 (shSTAT3) or scrambled control (shSCR), and cultured in semisolid medium + imatinib (1.0-2.5 $\mu$ M). STAT3 and not STAT5 knockdown was confirmed by immunoblot analyses (a, b, right). shSTAT3 reduced colony formation of K562 ${ }^{R}(\mathbf{a}$, left, $n=4)$ and AR230 ${ }^{R}$ (b, left, $n=4$ ) cells in the presence of imatinib, with no effect on parental TKI-sensitive controls. (c, d) TKI-resistant CML cell lines were transduced with dominant-negative STAT3 mutants (dnSTAT3) or empty vector (EV) and cultured in semisolid medium \pm imatinib $(1.0 \mu \mathrm{M})$. Inhibition of pSTAT3 ${ }^{\mathrm{Y} 705}$ was confirmed by immunoblot analyses (c, $\mathbf{d}$, right). dnSTAT3 reduced colony formation of K562 ${ }^{\mathrm{R}}$ (c, left,

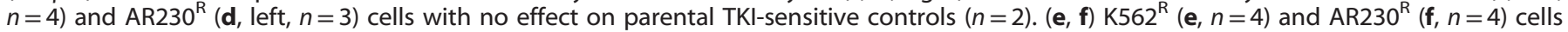
were incubated in methylcellulose semisolid medium with SF-1-066 (1-10 $\mu \mathrm{M}) \pm$ imatinib $(1.0 \mu \mathrm{M})$. SF-1-066 reduced colony formation of only TKI-resistant and not TKI-sensitive cells. (g) Mononuclear cells (MNCs) from peripheral blood of normal donors $(n=2)$ or CML ${ }^{\mathrm{CD} 34+}$ cells from newly diagnosed patients $(n=4)$ were treated ex vivo with SF-1-066 $(10 \mu \mathrm{M}) \pm$ imatinib $(2.5 \mu \mathrm{M})$ in RM or HS-5 CM for $96 \mathrm{~h}$ followed by colonyforming assays. All data are represented as percent of controls. Error bars represent s.e.m. ${ }^{*} P<0.05 ;{ }^{* *} P<0.01 ;{ }^{* * *} P<0.001$. 